\title{
Radical-Polar Crossover Reactions of Dienylboronate Complexes: Synthesis of Functionalized Allylboronic Esters
}

\author{
Marvin Kischkewitz, ${ }^{\dagger}$ Carolin Gerleve, ${ }^{\dagger}$ and Armido Studer*i) \\ Institute of Organic Chemistry, Westfälische Wilhelms-Universität, Corrensstraße 40, 48149 Münster, Germany
}

Supporting Information

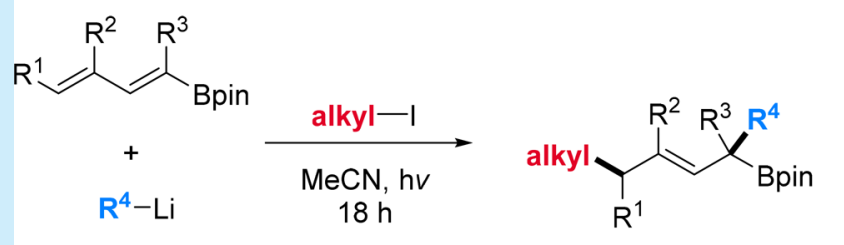

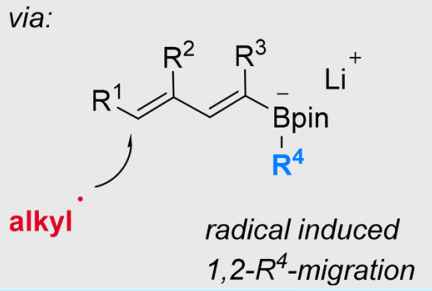

ABSTRACT: Radical-polar crossover reactions of dienylboronate complexes are applied to the synthesis of functionalized secondary and tertiary allylboronic esters. The transition-metal-free three-component coupling uses readily accessible dienylboronate esters as substrates in combination with various $\mathrm{sp}^{3} / \mathrm{sp}^{2}$ carbon nucleophiles and commercial alkyl iodides as radical precursors. In the visible light-initiated radical chain process, two new $\mathrm{C}-\mathrm{C}$ bonds are formed, and the $E$-double bond geometry in the product allylboronic esters is controlled with good to excellent selectivity.

A llylboronic esters are highly useful building blocks in organic synthesis. ${ }^{1}$ The great value of these reagents lies in their configurational and chemical stability. ${ }^{2}$ They have been successfully applied to cross-coupling reactions, ${ }^{3}$ but most often they are used as nucleophiles for the stereoselective synthesis of homoallylic secondary alcohols and amines by an allyl transfer reaction to carbonyls and imines. ${ }^{4}$ Notably, functionalized allylboronic esters can also participate in tandem reactions with aldehydes, ${ }^{5}$ and it was demonstrated that allylboronate complexes can react with a range of other types of carbon as well as heteroatom electrophiles with high $\gamma$-selectivity. ${ }^{6}$ Although various direct or indirect methods for the synthesis of allylboronic esters have been introduced, ${ }^{7}$ the development of novel strategies for their preparation is still of importance.

Along these lines, Morken and co-workers recently used bis(alkenyl)boronate complexes II in palladium-catalyzed three-component conjunctive cross-coupling reactions. ${ }^{8}$ Regioselective electrophilic aryl-palladation of the vinyl moiety induces a 1,2-alkenyl migration to provide, after reductive elimination, $\alpha$-arylmethyl allylboronic esters of type III (Scheme 1, a).

Our group and the Aggarwal laboratory have recently shown that electrophilic alkyl radicals add efficiently to in situ generated vinylboronate complexes $\mathbf{V}$ and that their corresponding radical anions undergo a radical polar crossover step, inducing a 1,2-R-migration to provide secondary and tertiary alkylboronic esters VI (Scheme 1, b). ${ }^{9,10}$ Motivated by these studies, we considered using dienylboronate complexes in radical-polar crossover reactions for the synthesis of $\alpha$ alkylated/arylated allylboronic esters. Importantly, the starting dienylboronic esters are readily accessible by hydrozirconation, ${ }^{11}$ boron-Wittig reaction ${ }^{12}$ or by Heck-Mizoroki cou-
Scheme 1. Synthesis of Allylboronic Esters in ThreeComponent Couplings

a) Conjunctive cross-coupling of bis(alkenyl)boronate complexes ${ }^{8}$

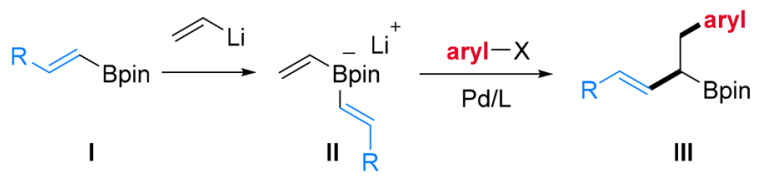

b) Radical induced 1,2-migration of vinylboronate complexes, ${ }^{9}$ 10a

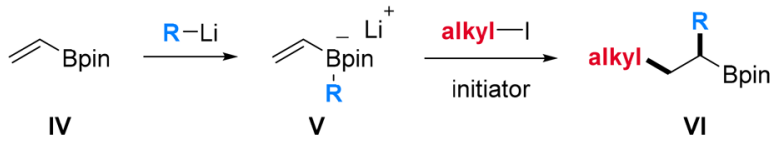

c) Radical induced 1,2-migration of dienylboronate complexes (this work)

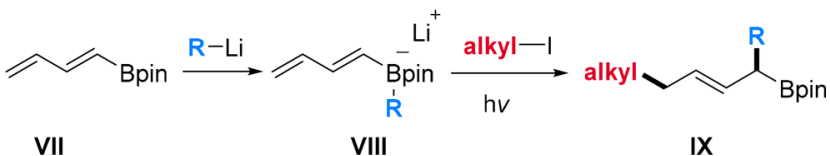

pling. ${ }^{13}$ We assumed that a carbon radical would selectively add to the $\delta$-position of the diene moiety in boronate complexes of type VIII (Scheme 1, c). The resulting allyl radical anion should then undergo a radical polar crossover step with concomitant 1,2 aryl- or alkyl-shift from boron to the $\alpha$-carbon. During the R-migration, the double bond geometry of the targeted allylboronic ester gets installed. A challenge will therefore lie in the control of the E/Z-selectivity. Moreover, for

Received: May 9, 2018

Published: June 6, 2018 
more complex substituted dienylboronate complexes, regioselective $\delta$-addition of the radical must be ensured. This novel transition-metal-free three-component strategy should enable the efficient construction of highly substituted and functionalized secondary and tertiary allylboronic esters IX.

We commenced our investigations by treating the readily prepared $\gamma$-methyl substituted dienylboronic ester 1a (see Supporting Information (SI)) with $n$-butyllithium at $0{ }^{\circ} \mathrm{C}$ in $\mathrm{Et}_{2} \mathrm{O}$ to generate the corresponding dienylboronate complex. After removal of the solvent, the crude ate complex was redissolved in acetonitrile, and 1.5 equiv of perfluorobutyl iodide was added. Visible light irradiation ${ }^{14}$ for $18 \mathrm{~h}$ afforded the desired perfluorinated allylboronic ester $2 a$, resulting from regioselective $\delta$-addition in $63 \%$ isolated yield with moderate 4:1 E/Z-selectivity (Table 1 , entry 1 ). The product derived

Table 1. Optimization Studies Using 1a as Substrate

\begin{tabular}{|c|c|c|c|c|}
\hline $1 a$ & 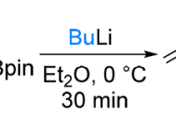 & $\underset{\substack{\text { Bu } \\
\text { Buin }}}{-\mathrm{Li}^{+}} \frac{\mathrm{C}_{4} \mathrm{~F}}{\mathrm{MeC}}$ & $\frac{(\text { equiv) }}{\stackrel{\mathrm{mL}), h v}{\mathrm{~h}}}$ & $\overbrace{\mathrm{Bp}}^{\mathrm{Bu}}$ \\
\hline entry $^{a}$ & C4F9I (equiv) & solvent $(\mathrm{mL})$ & yield $(\%)^{b}$ & $E / Z(\text { ratio })^{c}$ \\
\hline 1 & 1.5 & $\mathrm{MeCN}(2)$ & 63 & $4: 1$ \\
\hline 2 & 2.0 & $\mathrm{MeCN}(2)$ & 67 & $5: 1$ \\
\hline 3 & 5.0 & MeCN (2) & 69 & $9: 1$ \\
\hline 4 & 10.0 & $\mathrm{MeCN}(2)$ & 69 & $12: 1$ \\
\hline 5 & 5.0 & $\mathrm{MeCN}(1)$ & 66 & $8: 1$ \\
\hline
\end{tabular}

${ }^{a}$ Reactions were conducted with 1 a $(0.3 \mathrm{mmol}) .{ }^{b}$ Yields provided represent isolated yields. ${ }^{c}$ Selectivity was determined by ${ }^{1} \mathrm{H}$ NMR spectroscopy.

from $\beta$-addition was not identified. Increasing the amount of perfluoroalkyl iodide to 2.0 equiv resulted in a slightly higher yield $(67 \%)$ and enhanced selectivity (5:1, Table 1, entry 2 ). A further increase of the amount of perfluorobutyl iodide significantly affected the double bond selectivity without altering the yield. While using 5.0 equiv of iodide resulted in a 9:1 E/Z-selectivity, 10.0 equiv led to an even further improvement of the stereoselectivity to 12:1 (Table 1, entries 3 and 4). However, increasing the concentration of the reaction mixture did not affect the selectivity, but led to a slightly decreased yield (Table 1 , entry 5 ). Note that this positively influenced $E / Z$-selectivity, as a function of the amount of added radical precursor was found to be general and could be observed also in other transformations. Any time dependence of the $E / Z$-selectivity could be ruled out by varying the reaction time. Considering reaction economy and selectivity, we regarded 5.0 equiv of the radical precursor R-I as optimal (Table 1, entry 3). For comparative data on reactions conducted with 2.0 equiv of $\mathrm{R}-\mathrm{I}$ and varied reaction times, we refer to the SI.

To document the substrate scope, la was reacted under optimized conditions, varying the $\mathrm{C}$-nucleophile and also the radical precursor to give the allylboronic esters $\mathbf{2} \mathbf{b}-\mathbf{2 q}$ (Scheme 2 ). We first tested the ate complex derived from 1a and $n$ butyllithium in combination with various perfluoroalkyl iodides. With the exception of the volatile trifluoromethyl iodide, all other congeners performed well in this sequence, and the resulting perfluoroalkylated allylboronic esters $\mathbf{2 b} \mathbf{b} \mathbf{2 d}$ were isolated in good yields and good $E / Z$ selectivities. $\alpha$-Iodo ethyl esters are eligible $C$-radical precursors for this cascade, and higher $E / Z$ selectivity was achieved upon increasing the degree
Scheme 2. Radical-Polar Crossover Reaction of Various Dienylboronate Complexes ${ }^{a}$

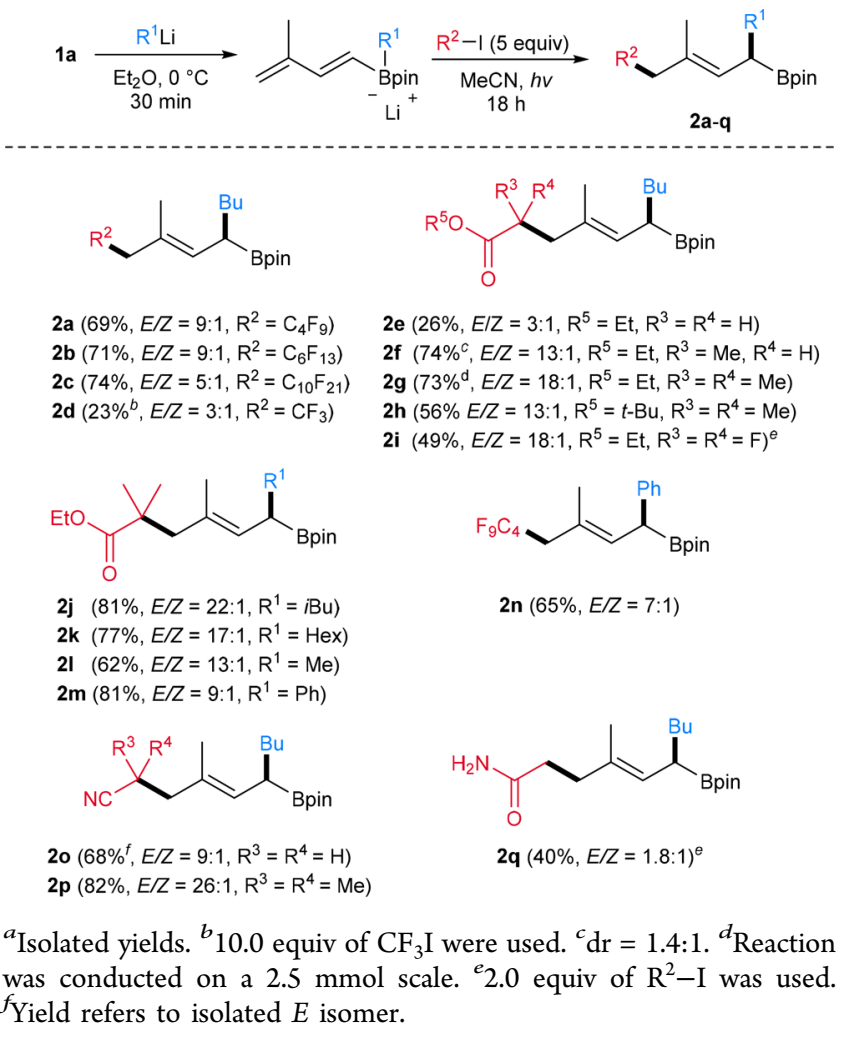

of $\alpha$-substitution in the ester moiety $(2 \mathbf{e}-\mathbf{2 g})$. As expected, low diastereoselectivity was noted for the reaction with $\alpha$-iodo ethyl propanoate. Replacing the ethyl by a tert-butyl ester group led to slightly lower $E / Z$-selectivity (2h). The $\alpha$-iodo- $\alpha$-difluorinated ester is a suitable $\mathrm{C}$-radical precursor, and $2 \mathbf{i}$ was isolated in good yield and high selectivity. The migrating alkyl group can be readily varied by replacing $n$-butyllithium with $i$-butyl-, $n$-hexyl-, and methyl-lithium $(2 \mathbf{j}-\mathbf{2 l})$. Importantly, the cascade also works efficiently for aryllithium reagents, as documented by the successful preparation of the secondary benzylic allylboronic esters $\mathbf{2 m}$ and $\mathbf{2 n}$. We next investigated $\alpha$-iodo acetonitrile and $\alpha$-iodo isobutyronitrile as radical precursors. Both nitriles worked well, and in analogy to the reaction with the $\alpha$-iodo esters, the $E / Z$ selectivity was increased by switching to the bulkier $\alpha$-dimethyl substituted system (from 9:1 to 26:1). Notably, unprotected $\alpha$-iodo amides are tolerated in the radicalpolar crossover reaction, as documented by the successful preparation of $\mathbf{2 q}$.

We next varied the dienylboronic ester moiety using nbutyllithium as the nucleophilic reaction component (Scheme 3). Starting with the unsubstituted boronic ester $\mathbf{1 b}$, perfluorobutyl iodide, $\alpha$-iodo isobutyronitrile, and ethyl dimethyl $\alpha$-iodo ester provided the targeted allylboronic esters $3 a-3 c$ in good yields and excellent $E / Z$ selectivities (up to $>99: 1$ ). The radical induced 1,2-migration also allows the introduction of secondary alkyl chains, as demonstrated by the successful preparation of the allylboronic ester 3d. Replacing the methyl with a $\gamma$-ethyl substituent at the diene acceptor leads to lower $E / Z$ selectivity, as shown for the synthesis of $\alpha$-iodo ester $3 \mathbf{e}$ and the nitrile analogue $3 \mathbf{f}$. Of note, $\gamma$-substituted tertiary allylboronic esters also could be accessed $(3 \mathbf{g}-\mathbf{3 j})$. We further demonstrated that the reaction also proceeds on a $\delta$ - 
Scheme 3. Radical-Polar Crossover Reaction of Dienylboronate Complexes: Variation of the Diene Moiety ${ }^{a}$

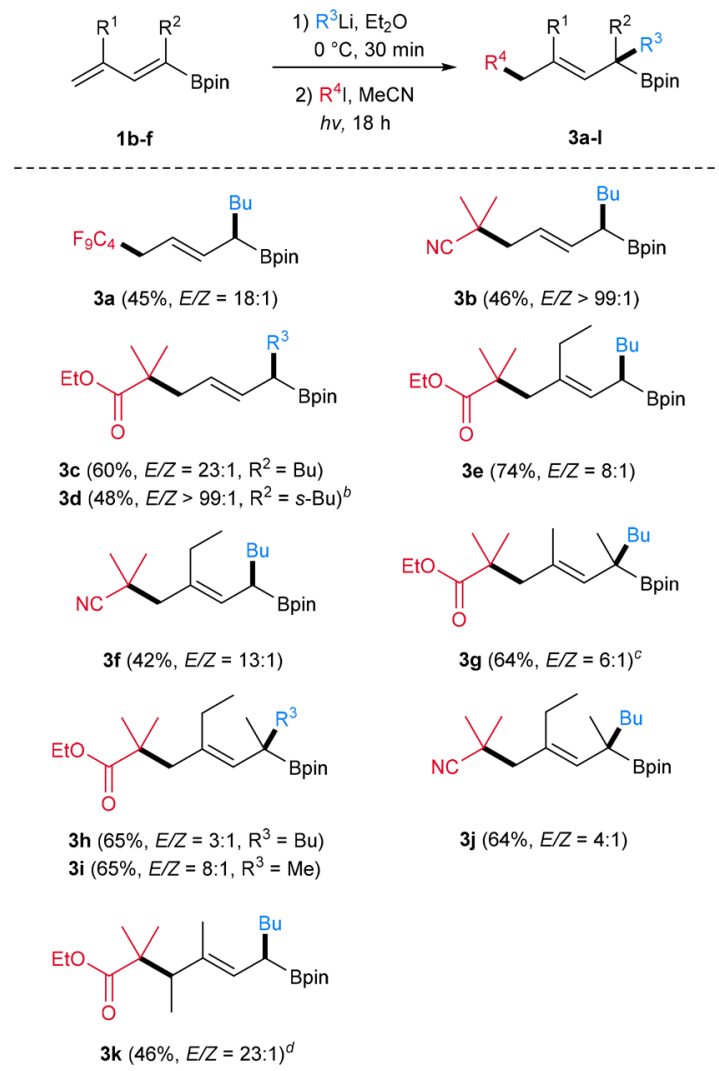

${ }^{a}$ Isolated yields. ${ }^{b} \mathrm{dr}=1.4: 1 .{ }^{c} 2.0$ equiv of $\mathrm{R}^{2}$-I was used. ${ }^{d} \mathrm{dr}=1.7: 1$.

substituted dienylboronate complex, and the resulting boronic ester $3 \mathbf{k}$ was isolated as a diastereomeric mixture in moderate yield and excellent $E / Z$ selectivity. The corresponding product resulting from $\beta$-addition was not identified showing the high intrinsic $\delta$-reactivity of these radical diene acceptors.

The suggested mechanism for the radical-polar crossover reaction is presented in Scheme 4. The cascade is initiated by

Scheme 4. Proposed Mechanism

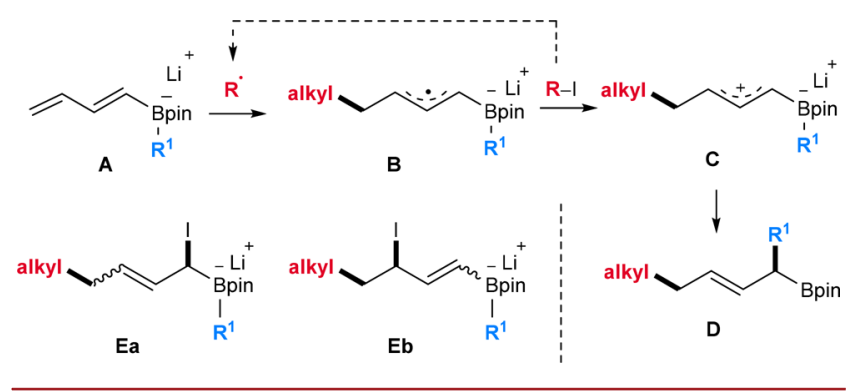

light-mediated C-I homolysis. The thus generated electrophilic $\mathrm{C}$-radical selectively adds to the $\delta$-position of the dienylboronate complex A, leading to the corresponding allyl radical anion B, which undergoes single electron oxidation by an alkyl iodide to generate the corresponding zwitterion $\mathbf{C}$. Thereby an alkyl radical is cogenerated, sustaining the radical chain. Hence, the radical cascade belongs to an electron-catalyzed process. ${ }^{15}$ The zwitterion $\mathbf{C}$ further reacts in an ionic 1,2-R-migration to provide the isolated allylboronic ester $\mathbf{D}$. Currently, we cannot fully exclude that the allyl radical anion $\mathbf{B}$ reacts via iodine atom abstraction from the alkyl iodide to generate atom-transfer products $\mathbf{E a}$ and/or $\mathbf{E b}$, which further react in a Matteson-type rearrangement ${ }^{16}$ to $D$. However, a mechanistic experiment, where the dienylboronate complex derived from $1 \mathbf{a}$ and $n$ butyllithium was treated with the Togni reagent, ${ }^{17}$ revealed formation of the trifluoromethylated allylboronic ester $\mathbf{2 d}$. Since this reagent cannot undergo an iodine transfer process, we regard the I atom-transfer/rearrangement sequence as less likely for these transformations.

To highlight the synthetic potential of the functionalized allylboron reagents, we conducted a series of allylation reactions (Scheme 5). The secondary allylboron reagents $2 \mathbf{h}$

Scheme 5. Diverse Functionalization of Allylboron Reagents
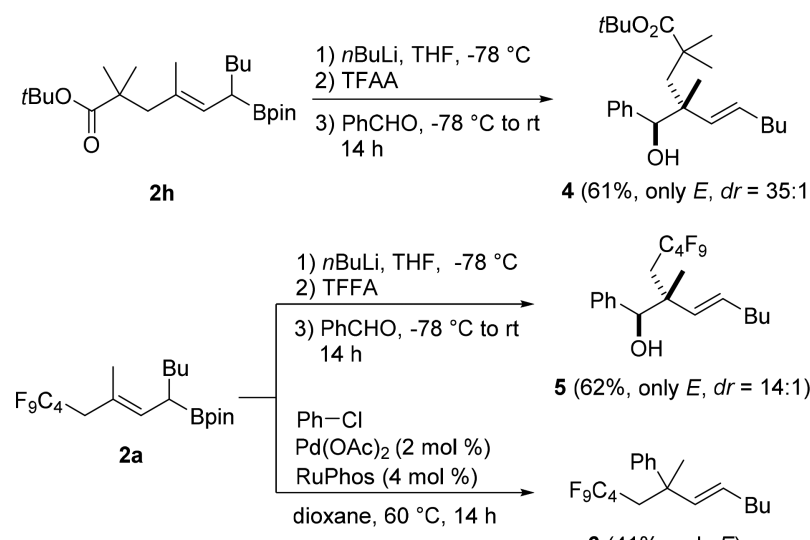

$4(61 \%$, only $E, d r=35: 1)$

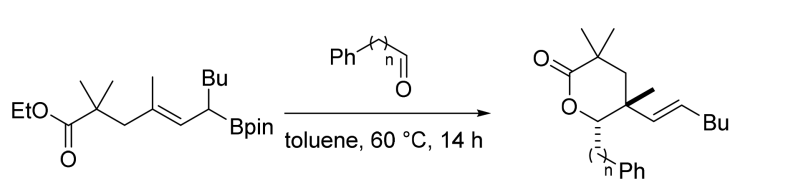

$2 \mathrm{~g}$

$7(56 \%, \mathrm{n}=0, E / Z=45: 1, d r=40: 1)$ $8(55 \%, \mathrm{n}=1 \text {, only } E, d r=12: 1)^{a}$

${ }^{a}$ In the presence of $10 \mathrm{~mol} \% \mathrm{Sc}(\mathrm{OTf})_{2}$.

and $\mathbf{2 a}$ were successfully used in highly diastereoselective benzaldehyde allylations ${ }^{18}$ (see 4 and 5). In addition, 2a was applied to a Pd-catalyzed $\gamma$-selective cross-coupling with chlorobenzene to give $6 .{ }^{19}$ To harvest the potential of the ethyl ester functional group, we used boronic ester $\mathbf{2 g}$ as substrate for a one-pot allylation/lactonization sequence ${ }^{20}$ and obtained 7 and $\mathbf{8}$ with excellent $E$ selectivity and very good diastereoselectivities. Hence, our method also represents a conceptually novel approach toward biologically valuable $\delta$ lactones.

In summary, we have developed a transition-metal-free threecomponent coupling of dienylboronic esters, alkyl/aryllithium compounds, and $\mathrm{R}-\mathrm{I}$ radical precursors for the synthesis of functionalized secondary and tertiary allylboronic esters with good to excellent $E / Z$ selectivity. The method is based on a visible light-initiated radical-polar crossover reaction of in situ generated dienylboronate complexes and works with a variety of commercial alkyl radical precursors.

\section{ASSOCIATED CONTENT}

\section{Supporting Information}

The Supporting Information is available free of charge on the ACS Publications website at DOI: 10.1021/acs.orglett.8b01459. 
Experimental details and characterization data for the starting material and products (PDF)

\section{AUTHOR INFORMATION}

\section{Corresponding Author}

*E-mail: studer@uni-muenster.de.

ORCID

Armido Studer: 0000-0002-1706-513X

\section{Author Contributions}

†These authors contributed equally.

Notes

The authors declare no competing financial interest.

\section{ACKNOWLEDGMENTS}

This work was supported by the Fonds der Chemischen Industrie (doctoral fellowship to C.G.) and the European Research Council (ERC Advanced grant agreement no. 692640).

\section{REFERENCES}

(1) Kennedy, J. W. J.; Hall, D. G. Angew. Chem., Int. Ed. 2003, 42, $4732-4739$

(2) Kramer, G. W.; Brown, H. C. J. Organomet. Chem. 1977, 132, 927.

(3) For selected methods, see: (a) Glasspoole, B. W.; Ghozati, K.; Moir, J. W.; Crudden, C. M. Chem. Commun. 2012, 48, 1230-1232. (b) Chausset-Boissarie, L.; Ghozati, K.; LaBine, E.; Chen, J. L.-Y.; Aggarwal, V. K.; Crudden, C. M. Chem. - Eur. J. 2013, 19, 1769817701. (c) Farmer, J. L.; Hunter, H. N.; Organ, M. G. J. Am. Chem. Soc. 2012, 134, 17470-17473. (d) Yang, Y.; Buchwald, S. L. J. Am. Chem. Soc. 2013, 135, 10642-10645. (e) Schuster, C. H.; Coombs, J. R.; Kasun, Z. A.; Morken, J. P. Org. Lett. 2014, 16, 4420-4423.

(f) Rybak, T.; Hall, D. G. Org. Lett. 2015, 17, 4156-4159.

(4) (a) Allylation of Carbonyl Compounds; Wiley: Hoboken, NJ, 2012.

(b) Yus, M.; González-Gómez, J. C.; Foubelo, F. Chem. Rev. 2013, 113, 5595-5698. (c) Denmark, S. E.; Fu, J. Chem. Rev. 2003, 103, 27632794.

(5) Kennedy, J. W. J.; Hall, D. G. Recent Advances in the Preparation of Allylboronates and Their Use in Tandem Reactions with Carbonyl Compounds; Wiley: Weinheim, 2005.

(6) García-Ruiz, C.; Chen, J. L.-Y.; Sandford, C.; Feeney, K.; Lorenzo, P.; Berionni, G.; Mayr, H.; Aggarwal, V. K. J. Am. Chem. Soc. 2017, 139, 15324-15327.

(7) For a recent review, see: Diner, C.; Szabó, K. J. J. Am. Chem. Soc. 2017, 139, 2-14.

(8) Edelstein, E. K.; Namirembe, S.; Morken, J. P. J. Am. Chem. Soc. 2017, 139, 5027-5030.

(9) Kischkewitz, M.; Okamoto, K.; Mück-Lichtenfeld, C.; Studer, A. Science 2017, 355, 936-938.

(10) (a) Silvi, M.; Sandford, C.; Aggarwal, V. K. J. Am. Chem. Soc. 2017, 139, 5736-5739. (b) Lovinger, G. J.; Morken, J. P. J. Am. Chem. Soc. 2017, 139, 17293-17296.

(11) Hart, D. W.; Blackburn, T. F.; Schwartz, J. J. Am. Chem. Soc. 1975, 97, 679-680.

(12) Coombs, J. R.; Zhang, L.; Morken, J. P. Org. Lett. 2015, 17, $1708-1711$.

(13) Madden, K. S.; David, S.; Knowles, J. P.; Whiting, A. Chem. Commun. 2015, 51, 11409-11412.

(14) Gerleve, C.; Kischkewitz, M.; Studer, A. Angew. Chem., Int. Ed. 2018, 57, 2441-2444.

(15) (a) Studer, A.; Curran, D. P. Nat. Chem. 2014, 6, 765-773. (b) Studer, A.; Curran, D. P. Angew. Chem., Int. Ed. 2016, 55, 58-102. (16) Matteson, D. S.; Mah, R. W. H. J. Am. Chem. Soc. 1963, 85, $2599-2603$.
(17) Charpentier, J.; Früh, N.; Togni, A. Chem. Rev. 2015, 115, 650682.

(18) Chen, J. L.-Y.; Scott, H. K.; Hesse, M. J.; Willis, C. L.; Aggarwal, V. K. J. Am. Chem. Soc. 2013, 135, 5316-5319.

(19) Potter, B.; Edelstein, E. K.; Morken, J. P. Org. Lett. 2016, 18, $3286-3289$.

(20) Kennedy, J. W. J.; Hall, D. G. J. Org. Chem. 2004, 69, 44124428. 